\title{
On the Study of Nonlinear Fractional Differential Equations on Unbounded Interval
}

\author{
Ahmed Hallaci ${ }^{1}$, Hamid Boulares ${ }^{\mp 2}$ and Muhammet Kurulay ${ }^{3}$ \\ 1,2 Depatement of Mathematics, Faculty of Sciences, University of 08 Mai 1945 Guelma, Guelma, Algeria \\ ${ }^{3}$ Yildiz Technical University, Department of Mathematics,Turkey \\ ${ }^{1}$ hal .ahmedguelm2@gmail.com, ${ }^{2}$ boulareshamid@gmail.com,${ }^{3}$ muhammetkurulay@gmail.com
}

\begin{abstract}
By the means of the variation of constants formula and some analytical skills, we use Banach contraction principle to investigate in this paper an uniqueness and existence of unbounded solution for nonlinear differential equations of fractional orders in weighted Banach space. At last, we present an illustrative example to support our main result.
\end{abstract}

Keywords: Fractional differential equations, Banach contraction principle, Existence and uniqueness. 2010 MSC No: 34A08, 47H10.

\section{Introduction}

The goal of this paper is to give sufficient conditions to show the existence and uniqueness of the following inital value problem of nonlinear fractional differential equations (FDEs)

$$
\left\{\begin{array}{c}
{ }^{C} D^{\alpha} u(t)=f(t, u(t)), t \geq 0, \\
u(0)=u_{0}, u^{\prime}(0)=u_{1},
\end{array}\right.
$$

where $1<\alpha<2, u_{0}, u_{1} \in \mathbb{R}, \mathbb{R}_{+}=[0,+\infty), f: \mathbb{R}_{+} \times \mathbb{R} \rightarrow \mathbb{R}$ is a continuous function, ${ }^{C} D^{\alpha}$ is the right side Caputo fractional derivative.

Recently, the subject of differential equations of fractional orders has become a fundamental field of applied mathematics due to Its ability to modeling of a lot of physical phenomena which associated to very rapid and very short changes as well as their importance in many mathematical models in science and engineering during the past three decades like physics, chemistry, biology, engineering, viscoelasticity, signal processing, electrotechnical, electrochemistry and controllability. Many researchers have been interested in the theory of nonlinear FDEs (see 10, 11, 13, 15]). But, the most of researches centred around the investigation on existence and uniqueness of solution where this side of study for nonlinear FDEs have been extensively developed using especially the fixed point theory and other theoretical methods as iterative method, measures of non-compactness technique, Krasnoselskii-Krein and nagumo uniqueness theorems (see [4, 16]). For example, the iterative medthod is employed in [16] to study the existence and uniqueness of solution for nonlinear neutral fractional integro-differential equation (FIDEs) of order $\alpha(0<\alpha<1)$. Farthermore, for the analytical solution of FDEs we refer 12 where the authors apply the Laplace transform for solving linear fractional-order differential equation.

However, the fixed point theorems staying the most used method to study the existence and uniqueness of solutions of nonlinear FDEs and nonlinear fractional differential systems, see [1]-[3], [5, 6, 9, 14, 17, 18]. In [1], the

\footnotetext{
${ }^{*}$ Corresponding author. Hamid Boulares ${ }^{1}$ boulareshamid@gmail.com
} 
Banach contraction principle and Krasnoselskii fixed point theorem are used with each other, to show the existence of the unique solution of nonlinear FDEs of order $\alpha(1<\alpha<2)$. As well, by utilizing the contraction mapping principle and the fixed point theorem due to O'Regan, the existence and uniqueness result for a FDEs is obtained in [2]. In [6], the authors discussed the existence and uniqueness of solution for some types of nonlocal fractional integro-differential equations which is based on Banach contraction principle. Farthermore, in [14 we find the applicability of Monch's fixed point theorem combined with Kuratowski measure of noncompactness for higher order FDEs to investigate the existence of unbounded nonnegative solutions in a weighted Banach space. Weighted Banach space is also employed in [18, where the authors presented an existence result of unique solution of FIDEs using the monotone iterative technique and the fixed point theory.

Motivated and inspired by the works mentioned above, we will use the Banach contraction principle to investigate the existence and uniqueness of the nonlinear FDEs of order $\alpha(1<\alpha<2)$ given by equations (1.1). For satisfy this aim, we first transform the system (1.1) into an ordinary differential equation of first-order and with a fractional integral term, then we use the means of the variation of constants formula, we obtain the equivalent integral equations of (1.1). After that, we prove the existence and uniqueness of nonlinear FDEs (1.1) by using the Banach fixed point theorem.

The rest of this paper is constructed under the following plan: in the next section, we give some concepts and lemmas of fractional calculus that will used to demonstrate our results. In Section 3, we present and demonstrate our main results. Lastly, an illustrative example to show the usefullness of our discussion is given.

\section{Preliminaries}

For mor detaits about the following important concepts of fractional calculus, see eg. [7, 8, 10, 13, 15] and the references therein.

Definition 2.1 ([13, 15]). The right side Riemman-Liouville fractional integral of order $\alpha>0$ of a function $u$ : $\mathbb{R}_{+} \rightarrow \mathbb{R}$ is defined by

$$
I^{\alpha} u(t)=\frac{1}{\Gamma(\alpha)} \int_{0}^{t}(t-s)^{\alpha-1} u(s) d s,
$$

where $\Gamma($.$) is the Gamma function given by the integral$

$$
\Gamma(\alpha)=\int_{0}^{\infty} e^{-\alpha} s^{\alpha-1} d s ; \alpha>0 .
$$

Definition $2.2([13,15])$. The right side Caputo fractional derivative of order $\alpha \in(n-1, n)$ of a function $u: \mathbb{R}_{+} \rightarrow \mathbb{R}^{-15}$ is defined by

$$
{ }^{C} D^{\alpha} u(t)=I^{n-\alpha} u^{(n)}(t)=\frac{1}{\Gamma(n-\alpha)} \int_{0}^{t}(t-s)^{n-\alpha-1} u^{(n)}(s) d s .
$$

Lemma 2.1 ([13, 15]). Let $\alpha>0$. Assume that $u \in C^{n-1}[0,+\infty)$, then

$$
\left(I^{\alpha C} D^{\alpha} u\right)(t)=u(t)-\sum_{j=0}^{n-1} \frac{u^{(j)}(0)}{j !} t^{j} .
$$

Remark 2.1. From Definitions 2.1, Definition 2.2 and Lemma 2.1, we get

(1) If $\alpha>0$ and $u \in C\left(\mathbb{R}_{+}\right)$, then ${ }^{C} D^{\alpha} I^{\alpha} u(t)=u(t)$, for all $t \in \mathbb{R}_{+}$.

(2) If $c$ is a constant real number, then ${ }^{C} D^{\alpha} c=0$, for all $\alpha>0$.

Now, we define the following Banach space which plays a fundamental role in our work.

Let $\rho: \mathbb{R}_{+} \rightarrow[1,+\infty)$ be a strictly nondecreasing continuous function with

$$
\rho(0)=1, \rho(t) \rightarrow \infty \text { as } t \rightarrow \infty, \rho(s) \rho(t-s) \leq \rho(t)
$$

for all $0 \leq s \leq t \leq \infty$. Let

$$
E=\left\{u \in C[0,+\infty): \sup _{t \geq 0}|u(t)| / \rho(t)<\infty\right\}
$$


Then $E$ is a Banach space endowed with the norm

$$
\|u\|=\sup _{t \geq 0} \frac{|u(t)|}{\rho(t)} .
$$

More properties of this Banach space are obtained in [7, 10. Farthermore, let

$$
\|\varphi\|_{t}=\max _{0 \leq s \leq t}|\varphi(s)|
$$

for any $t \geq 0$, any given $\varphi \in C\left(\mathbb{R}_{+}\right)$.

Lemma $2.2([13,15])$. Let $y \in C[0,+\infty)$. Then $u \in C[0,+\infty)$ is a solution of the initial value problem of fractional differential equations

$$
\left\{\begin{array}{c}
{ }^{C} D^{\alpha} u(t)=y(t), t \in \mathbb{R}_{+}, 1<\alpha<2, \\
u(0)=u_{0}, u^{\prime}(0)=u_{1}
\end{array}\right.
$$

if and only if $u$ is a solution of the initial value problem of first order ordinary differential equations

$$
\left\{\begin{array}{c}
u^{\prime}(t)=I_{0+}^{\alpha-1} y(t)+u_{1} \\
u(0)=u_{0}
\end{array}\right.
$$

Proof. We prove that for any $0<\beta<1$, if $\psi \in C[0,+\infty)$, then $\left(I^{\beta} \psi\right)(0)=0$. In fact, since

$$
I^{\beta} \psi(t)=\frac{1}{\Gamma(\beta)} \int_{0}^{t}(t-s)^{\beta-1} \psi(s) d s,
$$

we can conclude that

$$
\left|I^{\beta} \psi(t)\right|=\frac{1}{\Gamma(\beta)}\left|\int_{0}^{t}(t-s)^{\beta-1} \psi(s) d s\right| \leq \frac{\|\psi\|_{t}}{\Gamma(\beta+1)} t^{\beta} \rightarrow 0 \text { when } t \rightarrow 0 .
$$

(1) Let $u \in C[0,+\infty)$ be a solution of the fractional problem 2.1.

For any $t \in \mathbb{R}_{+}$, Definition 2.2 shows that

$$
{ }^{C} D^{\alpha} u(t)=\left({ }^{C} D^{\alpha-1} D^{1} u\right)(t)=y(t) .
$$

From Lemma 2.1, one gets

$$
u^{\prime}(t)=u^{\prime}(0)+I^{\alpha-1} r(t)=I^{\alpha-1} y(t)+u_{1},
$$

which means that $u$ is a solution of the problem 2.2 .

(2) Conversely, let $u$ be a solution of the problem 2.2 . For any $t \in \mathbb{R}_{+}$, taking into account Remark 2.1. we have

$$
{ }^{C} D^{\alpha} u(t)={ }^{C} D^{\alpha-1} u^{\prime}(t)=\left({ }^{C} D^{\alpha-1} I^{\alpha-1} y\right)(t)+{ }^{C} D^{\alpha-1} u_{1}=y(t) .
$$

Also, note that $y \in C[0,+\infty)$, we have $u^{\prime}(0)=I^{\alpha-1} y(0)+u_{1}=u_{1}$. Lemma 2.2 shows that the problem 1.1 is equivalent to the problem

$$
\left\{\begin{array}{c}
u^{\prime}(t)=\frac{1}{\Gamma(\alpha-1)} \int_{0}^{t}(t-s)^{\alpha-2} f(s, u(s)) d s+u_{1} \\
u(0)=u_{0}
\end{array}\right.
$$

Lemma 2.3. Let $k \in \mathbb{R}$ satisfies that

$$
e^{-k t} / \rho(t) \in B C[0,+\infty) \cap L^{1}[0,+\infty)
$$

Then (2.3) is equivalent to the following integral equation

$$
\begin{aligned}
u(t) & =u_{0} e^{-k t}+\frac{1-e^{-k t}}{k} u_{1}+k \int_{0}^{t} e^{-k(t-\tau)} u(\tau) d \tau \\
& +\frac{1}{\Gamma(\alpha-1)} \int_{0}^{t} \int_{\tau}^{t} e^{-k(t-s)}(s-\tau)^{\alpha-2} d s f(\tau, u(\tau)) d \tau
\end{aligned}
$$


Proof. It is clear that we can rewrite 2.3 as follow

$$
\left\{\begin{array}{c}
u^{\prime}(t)=-k u(t)+k u(t)+\frac{1}{\Gamma(\alpha-1)} \int_{0}^{t}(t-s)^{\alpha-2} f(s, u(s)) d s+u_{1}, \\
u(0)=u_{0},
\end{array}\right.
$$

or in other term

$$
u^{\prime}(t)+k u(t)=k u(t)+\frac{1}{\Gamma(\alpha-1)} \int_{0}^{t}(t-s)^{\alpha-2} f(s, u(s)) d s+u_{1}, u(0)=u_{0} .
$$

We use the variation of constants formula to get

$$
\begin{aligned}
u(t) & =u_{0} e^{-k t}+e^{-k t} \int_{0}^{t}\left[k u(s)+\frac{1}{\Gamma(\alpha-1)} \int_{0}^{s}(s-\tau)^{\alpha-2} f(\tau, u(\tau)) d \tau+u_{1}\right] e^{k s} d s \\
& =u_{0} e^{-k t}+e^{-k t} \int_{0}^{t} k u(s) e^{k s} d s+\frac{1}{\Gamma(\alpha-1)} \int_{0}^{t} \int_{0}^{s} e^{-k(t-s)}(s-\tau)^{\alpha-2} f(\tau, u(\tau)) d s d \tau \\
& +u_{1} e^{-k t} \int_{0}^{t} e^{k s} d s \\
& =u_{0} e^{-k t}+k \int_{0}^{t} e^{-k(t-s)} u(s) d s+\frac{1}{\Gamma(\alpha-1)} \int_{0}^{t} \int_{0}^{s} e^{-k(t-s)}(s-\tau)^{\alpha-2} f(\tau, u(\tau)) d s d \tau \\
& +u_{1} e^{-k t} \int_{0}^{t} e^{k s} d s \\
& =u_{0} e^{-k t}+\frac{1-e^{-k t}}{k} u_{1}+k \int_{0}^{t} e^{-k(t-s)} u(s) d s \\
& +\frac{1}{\Gamma(\alpha-1)} \int_{0}^{t} \int_{\tau}^{t} e^{-k(t-s)}(s-\tau)^{\alpha-2} d s f(\tau, u(\tau)) d \tau .
\end{aligned}
$$

Farthermore, it is clear that

$$
\left(e^{k t} u(t)\right)^{\prime}=\left(u^{\prime}(t)+k u(t)\right) e^{k t}
$$

using this fact we get

$$
\begin{aligned}
\left(u^{\prime}(t)+k u(t)\right) e^{k t}= & {\left[u_{0}+\frac{e^{k t}-1}{k} u_{1}+k \int_{0}^{t} e^{k s} u(s) d s\right.} \\
& \left.+\frac{1}{\Gamma(\alpha-1)} \int_{0}^{t} \int_{\tau}^{t} e^{k s}(s-\tau)^{\alpha-2} d s f(\tau, u(\tau)) d \tau\right] \\
= & e^{k t} u_{1}+k e^{k t} u(t)+\left[\int_{0}^{t} e^{k \tau} I_{0^{+}}^{\alpha-1} f(\tau, u(\tau)) d \tau\right] \\
= & e^{k t}\left(u_{1}+I_{0^{+}}^{\alpha-1} f(t, u(t))+k u(t)\right) .
\end{aligned}
$$

Beside, if

$$
\begin{aligned}
u(t)= & u_{0} e^{-k t}+\frac{1-e^{-k t}}{k} u_{1}+k \int_{0}^{t} e^{-k(t-s)} u(s) d s \\
& +\frac{1}{\Gamma(\alpha-1)} \int_{0}^{t} \int_{\tau}^{t} e^{-k(t-s)}(s-\tau)^{\alpha-2} d s f(\tau, u(\tau)) d \tau
\end{aligned}
$$

holds we have $u(0)=u_{0}$. This achieves the proof.

The following fixed point theorem is needed to prove our main result.

Lemma 2.4. [8] (Banach contraction principle) Let $E$ be a Banach space and $A: E \rightarrow E$ an operator. If $A$ is a contraction, then $A$ has a unique fixed point. 


\section{Main results}

We define the operator $A: E \longrightarrow E$ by

$$
\begin{aligned}
A u(t) & =u_{0} e^{-k t}+\frac{1-e^{-k t}}{k} u_{1}+k \int_{0}^{t} e^{-k(t-\tau)} u(\tau) d \tau \\
& +\frac{1}{\Gamma(\alpha-1)} \int_{0}^{t} \int_{\tau}^{t} e^{-k(t-s)}(s-\tau)^{\alpha-2} d s f(\tau, u(\tau)) d \tau
\end{aligned}
$$

for all $t \in \mathbb{R}_{+}$and $k \in \mathbb{R}$.

In this section, we will be interested in studying the existence and uniqueness of fixed point of the operator $A$ which represent the unique solution of system (1.1).

Theorem 3.1. Assume that hypothesis (2.4) holds and

$(H)$ There exists a constant $l>0$ and a function $\Phi:[0, \infty) \rightarrow[0, \infty)$ so that if $|u|,|v| \leq l$ then

$$
|f(t, u)-f(t, v)| \leq \Phi(t)|u-v|
$$

for all $t \in \mathbb{R}_{+}$, and

(ii) There exists a constant $c \in(0,1)$ which satisfies

$$
|k| \int_{0}^{\infty} \frac{e^{-k t}}{\rho(t)} d t+\sup _{t \geq 0} \int_{0}^{t} \frac{K(t-\tau)}{\rho(t-\tau)} \Phi(\tau) d \tau \leq c,
$$

where

$$
K(t-\tau)= \begin{cases}\frac{1}{\Gamma(\alpha-1)} \int_{\tau}^{t} e^{-k(t-s)}(s-\tau)^{\alpha-2} d s, & t \geq \tau \\ 0, & t \leq \tau\end{cases}
$$

Then the system (1.1) admits a unique solution in Banach space E.

Proof. We show that $A: E \rightarrow E$ is a contraction mapping. Indeed, let $u, v \in E, t \geq 0$ and due to 3.2 , we have

$$
\begin{aligned}
& \left|\frac{A u(t)}{h(t)}-\frac{A v(t)}{h(t)}\right| \\
& =\left|k \int_{0}^{t} \frac{e^{-k(t-\tau)}}{\rho(t)}[u(\tau)-v(\tau)] d \tau+\int_{0}^{t} \frac{K(t-\tau)}{\rho(t)}[f(\tau, u(\tau))-f(\tau, v(\tau))] d \tau\right| \\
& \leq|k| \int_{0}^{t} \frac{e^{-k(t-\tau)}}{\rho(t-\tau)} \frac{|u(\tau)-v(\tau)|}{\rho(\tau)} d u+\int_{0}^{t} \frac{K(t-\tau)}{\rho(t-\tau)} \frac{|f(\tau, u(\tau))-f(\tau, v(\tau))|}{\rho(\tau)} d \tau \\
& \leq|k| \int_{0}^{t} \frac{e^{-k(t-\tau)}}{\rho(t-v)} \frac{|u(\tau)-v(\tau)|}{\rho(\tau)} d \tau+\int_{0}^{t} \frac{K(t-\tau)}{\rho(t-\tau)} \Phi(\tau) \frac{|u(\tau)-v(\tau)|}{\rho(\tau)} d \tau \\
& \leq|k| \sup _{t \geq 0}\left[\frac{|u(\tau)-v(\tau)|}{\rho(\tau)}\right] \int_{0}^{t} \frac{e^{-k(t-\tau)}}{\rho(t-\tau)} d \tau+\sup \left[\frac{|u(\tau)-v(\tau)|]}{\rho(\tau)}\right] \int_{0}^{t} \frac{K(t-\tau)}{\rho(t-\tau)} \Phi(\tau) d \tau \\
& \leq|k|\|u-y\| \int_{0}^{t} \frac{e^{-k(t-\tau)}}{\rho(t-\tau)} d u+\|u-v\| \int_{0}^{t} \frac{K(t-\tau)}{\rho(t-\tau)} \Phi(\tau) d \tau \\
& \leq\left[|k| \int_{0}^{t} \frac{e^{-k(t-\tau)}}{\rho(t-\tau)} d \tau+\int_{0}^{t} \frac{K(t-\tau)}{\rho(t-\tau)} \Phi(\tau) d \tau\right]\|u-v\|,
\end{aligned}
$$

taking into account 2.4 and $(3.3)$, we get

$$
\|A u-A v\| \leq c\|u-v\|
$$

so, $A$ is a contraction mapping from $E$ into $E$.

Hence, using the Banach contraction principle and from Theorem 3.1, the operator $A$ given by (3.1) has a unique fixed point which represent the unique solution of system 1.1. 


\section{Example}

Consider the following initial value problem of Caputo type fractional differential equations

$$
\left\{\begin{array}{c}
{ }^{C} D_{0+}^{\frac{3}{2}} u(t)=\omega(t)\left(\frac{1+\alpha u+\sin u}{e^{\epsilon t}}\right), \\
u(0)=x_{0}, u^{\prime}(0)=u_{1},
\end{array}\right.
$$

where $\omega(t)=\epsilon^{\frac{-1}{2}} \frac{1}{\epsilon+t^{2}}, \epsilon>0, \alpha>-1, \lambda>1, k \in \mathbb{R}, t \geq 0$. Suppose $0<|k| \leq \frac{\lambda-1}{2}$ and let $\rho(t)=e^{\lambda t}$, clearly that 2.4 holds and $|k| \int_{0}^{\infty} \frac{e^{-k t}}{\rho(t)} d t \leq \frac{|k|}{\lambda+k}$, then we define the Banach space by

$$
E_{\lambda}=\left\{u(t) \in C[0,+\infty): \sup _{t \geq 0} \frac{|u(t)|}{e^{\lambda t}}<\infty\right\},
$$

equipped with the norm $\|u\|=\sup _{t \geq 0} \frac{|u(t)|}{e^{\lambda t}}$. Clearly

$$
|f(t, u)-f(t, v)| \leq \Phi(t)|u-v|
$$

where $\Phi(t)=(1+\alpha) \omega(t)$ for all $t \geq 0$.

$$
\begin{aligned}
\frac{K(t-\tau)}{e^{\lambda(t-\tau)}} & =\frac{1}{\Gamma(1 / 2)} \int_{\tau}^{t} \frac{1}{e^{(\lambda+k)(t-s)}} \frac{(s-\tau)^{-1 / 2}}{e^{\lambda(s-\tau)}} d s \\
& \leq \frac{\int_{\tau}^{t} \frac{(s-\tau)^{-1 / 2}}{e^{\lambda(s-\tau)}} d s}{\Gamma(1 / 2)}=\frac{\int_{0}^{t-\tau} \frac{\tau^{-1 / 2}}{e^{\lambda \tau}} d \tau}{\Gamma(1 / 2)} \leq \lambda^{1 / 2}
\end{aligned}
$$

for all $t \geq 0$.

Moreover, if we choose $\epsilon \geq(1+\alpha) \lambda^{1 / 2} \pi(\lambda+k)$ then for all $t \geq 0$, we get

$$
\begin{aligned}
\int_{0}^{t} \frac{K(t-\tau)}{h(t-\tau)} \Phi(\tau) d \tau & =(1+\alpha) \lambda^{1 / 2} \epsilon^{\frac{-1}{2}} \int_{0}^{t} \frac{1}{\epsilon+\tau^{2}} d \tau \\
& =(1+\alpha) \lambda^{1 / 2} \epsilon^{\frac{-3}{2}} \int_{0}^{t} \frac{1}{1+\left(\tau \epsilon^{\frac{-1}{2}}\right)^{2}} d \tau \\
& =(1+\alpha) \lambda^{1 / 2} \epsilon^{-1} \int_{0}^{t \epsilon^{\frac{-1}{2}}} \frac{d z}{1+z^{2}} \\
& =(1+\alpha) \lambda^{1 / 2} \epsilon^{-1} \arctan \left(t \epsilon^{\frac{-1}{2}}\right) \\
& \leq(1+\alpha) \lambda^{1 / 2} \epsilon^{-1} \frac{\pi}{2} \\
& \leq \frac{1}{2(\lambda+k)}<1-\frac{|k|}{\lambda+k}
\end{aligned}
$$

Then there exists $c=\frac{|k|}{\lambda+k}+\frac{1}{2(\lambda+k)}<1$ which satisfies $\|A u-A v\| \leq c\|u-v\|$.

So, the system 4.1 possesses a unique solution in $E_{\lambda}$ follows from Theorem 3.1 .

\section{References}

[1] B. Ahmad, J. J. Nieto, Existence Results for Nonlinear Boundary Value Problems of Fractional Integrodifferential Equations with Integral Boundary Conditions, Hindawi Publishing Corporation, Boundary Value Problems, 2009(1) Article ID 708576,11pages, doi:10.1155/2009/708576. https://doi.org/10.1155/2009/708576

[2] B. Ahmad, S. K. Ntouyas, A. Alsaedi and H. Al-Hutami, Nonlinear q-fractional differential equations with nonlocal and sub-strip type boundary conditions, Electron. J. Qual. Theory Differ. Equ. 26(2014) (2014) 1-12. https://doi.org/10.14232/ejqtde.2014.1.26 
[3] B. Ahmad, S. Sivasundaram, On four-point nonlocal boundary value problems of nonlinear integrodifferential equations of fractional order, Applied Mathematics and Computation 217(2) (2010) $480-487$. https://doi.org/10.1016/j.amc.2010.05.080

[4] A. Anguraj and P. Karthikeyan. Existence of solutions for nonlocal semilinear fractional integro-differential equation with KrasnoselskiiKrein-type conditions. Nonlinear Stud., 19(3) (2012) 433-442.

[5] A. Anguraj, P. Karthikeyan, M. Rivero, J.J. Trujillo, On new existence results for fractional integro-differential equations with impulsive and integral conditions, Computers and Mathematics with Applications 66 (2014) 2587-2594.https://doi.org/10.1016/j.camwa.2013.01.034

[6] K. Balachandran, J. J. Trujillo, The nonlocal Cauchy problem for nonlinear fractional integrodifferential equations in Banach spaces, Nonlinear Analysis 72 (2010) 4587-4593.https://doi.org/10.1016/j.na.2010.02.035

[7] T.A. Burton, Stability by Fixed Point Theory for Functional Differential Equations, Dover publications INC, Mineola, New York, 2006.

[8] T.A. Burton, Stability and Periodic Solutions of Ordinary and Functional Differential Equations, Academic Press, INC. Orlando, Florida, 1985.https://doi.org/10.1016/s0076-5392(09)x6019-4

[9] D. N. Chalishajar, Existence and uniqueness results for boundary value problems of higher order fractional integro-differential equations involving gronwall's inequality in banach spaces. Acta Mathematica Scientia, 33(3) (2013) 758-772.https://doi.org/10.1016/s0252-9602(13)60036-3

[10] S. Das, Functional Fractional Calculus. Springer science and business media, 2011.

[11] R., Hilfer. Application of fractional calculus in physics, pp. 699-707.World Scientific, Singapore, 2000.

[12] S. Kazem, Exact Solution of Some Linear Fractional Differential Equations by Laplace Transform, International Journal of Nonlinear Science, Vol.16(1) (2013), 3-11.

[13] A. A. Kilbas, H.M. Srivastava, J. J. Trujillo, Theory and Applications of Fractional Differential Equations, Elsevier, 2006.https://doi.org/10.1016/s0304-0208(06)x8001-5

[14] Z. Liu, L. Liu, Y. Wu, J. Zhao, Unbounded Solutions of a Boundary Value Problem for Abstractnth-Order Differential Equations on an Infinite Interval, Hindawi Publishing Corporation, Journal of Applied Mathematics and Stochastic Analysis, Volume 2008, Article ID 589480,11pages, doi:10.1155/2008/589480.

[15] I. Podlubny, Fractional Differential Equations, Academic Press, San Diego, 1999.

[16] G. Wang, S. Liu, L. Zhang, Neutral fractional integro-differential equation with nonlinear term depending on lower order derivative, Journal of Computational and Applied Mathematics, 260 (2014) 167172.https://doi.org/10.1016/j.cam.2013.09.051

[17] J. Wang, Y. Yang, W. Wei, Nonlocal impulsive problems for fractional differential equations with time-varying generating operators in Banach spaces, Opuscula Mathematica, 30(3) (2010) 361381.https://doi.org/10.7494/opmath.2010.30.3.361

[18] L. Zhang, B. Ahmad, G. Wang, R. P. Agarwal, Nonlinear fractional integro-differential equations on unbounded domains in a Banach space, Journal of Computational and Applied Mathematics 249 (2013) 5156.https://doi.org/10.1016/j.cam.2013.02.010 